\title{
UNA DÉCADA DE ALTAS MÉDICAS NO EFECTIVIZADAS EN UN HOSPITAL DE LIMA, PERÚ
}

\author{
Félix García ${ }^{1,2, a}$, Javier Cieza ${ }^{1,2, b}$, Jorge Flores ${ }^{1, c}$, Julio Huapaya ${ }^{3, d}$, Ana Obregón $n^{2, d}$
}

\begin{abstract}
RESUMEN
El objetivo del estudio fue describir los cambios financieros de los días de alta no efectiva desde el año 2001 al 2010 , en un hospital general de Lima Metropolitana. Para ello, se obtuvieron los montos totales pagados y exonerados de las cuentas de los pacientes con alta médica no efectivizada, por falta de pago del hospital general. Encontramos que el número de pacientes con alta médica no efectivizada por falta de pago disminuyó del 2001 al 2010 en 77\%, los días desde el alta médica hasta que el paciente abandonó el hospital disminuyeron en $80 \%$; las cuentas totales, pagadas y exoneradas, se redujeron en 63,53 y $68 \%$ respectivamente. Asimismo, el porcentaje promedio exonerado a cada paciente con alta médica no efectivizada por falta de pago, en la década estudiada, fue $61,7 \%$. En conclusión, el alta no efectiva incrementa los costos al paciente, teniendo que ser asumido por el hospital al exonerar la cuenta, aunque este gasto disminuyó en el periodo estudiado, esto podría deberse a la implementación de políticas de aseguramiento en salud.
\end{abstract}

Palabras clave: Inversiones en salud; Gastos en salud; Costos de la atención en salud; Alta del paciente; Hospitales; Costos de la atención en salud (fuente: DeCS BIREME).

\section{A DECADE OF NO EFFECTIVE INPATIENT MEDICAL DISCHARGES, EXPERIENCE FROM A HOSPITAL IN LIMA, PERU}

\begin{abstract}
Our aim is to describe the financial implications of no effective in patient discharges from 2001-2010 from a general hospital in lima city. For this purpose we analyzed the total amounts, cancellations and exonarations from the patient accounts with a non effective medical discharge because of hospital "debts". We found that the number of patients with a non effective medical discharge decreased $70 \%$ from 2001 to 2010 , the number of days between the medical discharge until the day the patient left the hospital decreased $80 \%$. The total amounts, cancellations and exonarations decreased $63 \%, 53 \%$ and $68 \%$, respectively. The average amount of exoneration was $61,7 \%$. In conclusion, the non effective medical discharges increase patient debts, which are partially exonerated and assumed by the hospital. Even though it has decreased in the last decade, this could be explained by the implementation of the new health insurance policies.
\end{abstract}

Key words: Investments in health; Health Expenditures; Health Care Costs; Patient discharge; Hospitals; Health care costs (source: MeSH NLM).

\section{INTRODUCCIÓN}

El desarrollo de los países puede medirse bajo muchos puntos de vista. Sin embargo, algunos aspectos relacionados con la relevancia del ciudadano para un estado estructurado son la educación y la salud. Sobre este último aspecto, la Constitución Política del Perú de 1993 estableció que todos tienen derecho a la protección de su salud ${ }^{(1)}$.

Asimismo, la salud puede traducirse cuantificando el gasto en salud per cápita (GSPC) o en función del Producto Nacional Bruto (PNB). Los países industrializados tienen un GSPC de USD 4590 por año o, visto como gasto en salud, en función del PNB de 11,1\%. En América del Sur, países como Chile, Brasil y Argentina tienen un GSPC de USD 762; USD 721, y USD 610 y como porcentaje del PNB de 7,5; 8,4 y 7,4\% respectivamente (2).

El Perú tiene un GSPC de USD 200 y un gasto en salud en función del PNB de $4,5 \%$, datos que reflejan un déficit en inversión en salud comparado con países cercanos, como los mencionados anteriormente. De alguna manera, estos datos son también claros indicadores de una falta de inversión en la recuperación de la salud

${ }^{1}$ Hospital Nacional Cayetano Heredia. Lima, Perú.

${ }^{2}$ Facultad de Medicina Alberto Hurtado, Universidad Peruana Cayetano Heredia. Lima, Perú.

${ }^{3}$ Sociedad Científica de Estudiantes de Medicina, Universidad Peruana Cayetano Heredia. Lima, Perú.

${ }^{\mathrm{a}}$ Médico magíster en Educación con mención en Docencia e Investigación; ${ }^{\mathrm{b}}$ médico doctor en Medicina; ${ }^{\mathrm{c}}$ médico cirujano; ${ }^{\mathrm{d}}$ estudiante de Medicina

Recibido: 16-04-12 Aprobado: 27-06-12 
perdida, situación grave en cualquier país, pero mucho más grave en un país cuyo Producto Bruto Interno $(\mathrm{PBI})$ es relativamente pequeño comparado, por ejemplo, con Chile (80\%), Colombia (56\%) o Argentina (42\%), resultado de una gran cantidad de ciudadanos pobres o muy pobres ${ }^{(3)}$.

En consecuencia, es relevante conocer el impacto de normativas que afectan a las instituciones dedicadas a la recuperación de la salud como los hospitales, dado que evaluar estas normativas directamente en la población puede ser altamente dificultoso si se quieren obtener datos objetivos y precisos. Esta información es casi nula, inexistente o muy atomizada en nuestro país y no permite tener una idea clara del efecto de la toma de decisiones políticas de cierta envergadura en los hospitales nacionales administrados por el Ministerio de Salud (MINSA), principal medio de acceso a servicios de recuperación de la salud en el Perú (4).

Adicionalmente, es oportuno iniciar un proceso de evaluación constante de la toma de decisiones, sobre todo en los hospitales del MINSA, pues ellos ofertan servicios a la gran mayoría de peruanos y, en especial, a los sectores más desprotegidos social y económicamente. Esto adquiere una mayor relevancia cuando el crecimiento del $\mathrm{PBI}$ del Perú no parece traducirse en mejor atención a estos sectores. Estudios como el presente pueden dar inicio a una discusión más objetiva de los gastos en salud.

Por lo expresado, este estudio tuvo como objetivo realizar una descripción de los cambios financieros entre el año 2001 y el 2010 sobre un hospital general de Lima Metropolitana ubicado en el sector norte de la ciudad de Lima Metropolitana, debido a que estos cambios podrían deberse a las directivas implementadas en el sistema integral de aseguramiento público de salud, pues el hospital tiene un área de influencia mediata de casi tres millones de habitantes, en su mayoría ciudadanos pobres o extremadamente pobres, principales beneficiaros del sistema de aseguramiento.

\section{EL ESTUDIO}

El presente es un estudio descriptivo cuya población estuvo conformada por todos los pacientes con alta médica no efectivizada por falta de pago del Hospital Nacional Cayetano Heredia $(\mathrm{HNCH})$, entre los años 2001 y 2010. Se utilizó como fuentes de información secundaria los informes del Departamento de Servicio Social, los que contienen el número de pacientes; el número de días de internamiento innecesarios posteriores al alta médica, y la cuenta total, pagada y exonerada, de los pacientes con alta médica no efectivizada por falta de pago.

Las variables se definieron operacionalmente como número de egresos hospitalarios: personas retiradas de los servicios de hospitalización; número de pacientes de alta médica no efectivizada: personas que después de indicada el alta médica permanecieron hospitalizadas por falta de pago; número de días postalta médica: días transcurridos desde el alta médica hasta que el paciente abandona el hospital; cuenta pagada: dinero que el paciente abona al abandonar el hospital por los servicios no pagados al momento de recibirlos; cuenta exonerada: dinero que el paciente "no abona" al abandonar el hospital por los servicios recibidos; cuenta total: es la suma de la cuenta pagada y la cuenta exonerada; cuenta total promedio por paciente de alta médica no efectivizada: [cuenta total/número de pacientes]; cuenta pagada promedio por paciente de alta médica no efectivizada: [cuenta pagada/número de pacientes]; cuenta exonerada promedio por paciente de alta médica no efectivizada: [cuenta exoneradal número de pacientes]; porcentaje de cuenta exonerada: [(cuenta exonerada/cuenta total) x 100]

Para los cálculos monetarios, se transformaron los nuevos soles peruanos en dólares americanos (USD). Para ello, se utilizó el tipo de cambio promedio en los bancos del mes de junio del correspondiente año según información de la Superintendencia de Banca y Seguros del Perú.

Los datos obtenidos sirvieron para cumplir con el objetivo del estudio ya que muestran cambios financieros del $\mathrm{HNCH}$ en una década, en la que se implementaron una serie de dispositivos tendientes a incrementar el número de beneficiarios del Seguro Integral de Salud (SIS).

\section{HALLAZGOS}

La variación de las variables estudiadas sobre costos hospitalarios y por paciente del alta médica no efectivizada, del año 2001 al año 2010 se muestran en la Tabla 1.

El número de pacientes con alta médica no efectivizada por falta de pago disminuyó del año 2001 al año 2010 en $77 \%$. Los días desde el alta médica hasta que el paciente abandonó el hospital disminuyeron en $80 \%$. Las cuentas totales, pagadas y exoneradas, de los pacientes con alta médica no efectivizada por falta de pago también disminuyeron en el periodo estudiado en 63,53 y $68 \%$ respectivamente. 
Tabla 1. Variación de los costos hospitalarios y por paciente de alta médica no efectivizada, entre los años 2001 y 2010.

\begin{tabular}{|c|c|c|c|c|c|c|c|c|c|c|}
\hline & 2001 & 2002 & 2003 & 2004 & 2005 & 2006 & 2007 & 2008 & 2009 & 2010 \\
\hline $\mathrm{N} .^{\circ}$ egresos hospitalarios & 12878 & 16065 & 17768 & 18985 & 17913 & 17018 & 18193 & 19373 & 16563 & 17279 \\
\hline $\begin{array}{l}\mathrm{N} .^{\circ} \text { pacientes de alta médica no } \\
\text { efectivizada }\end{array}$ & 2120 & 1102 & 881 & 914 & 833 & 733 & 513 & 357 & 321 & 492 \\
\hline N. ${ }^{\circ}$ días postalta médica ${ }^{a}$ & 6871 & 3017 & 2209 & 2645 & 2234 & 1983 & 1331 & 717 & 918 & 1371 \\
\hline \multicolumn{11}{|l|}{ Cuenta hospitalaria postalta (USD) * } \\
\hline Cuenta pagada & 179224 & 144740 & 89460 & 117545 & 97955 & 96950 & 62900 & 42105 & 29479 & 83385 \\
\hline Cuenta exonerada & 359467 & 153341 & 173304 & 212784 & 174810 & 151435 & 91570 & 77868 & 46193 & 114916 \\
\hline Cuenta total ${ }^{\mathrm{b}}$ & 538692 & 298081 & 262765 & 330330 & 272765 & 248385 & 154470 & 119973 & 75672 & 198302 \\
\hline \multicolumn{11}{|c|}{ Cuenta postalta promedio por paciente (USD) * } \\
\hline Cuenta pagada & 84,5 & 131,3 & 101,5 & 128,6 & 117,6 & 132,3 & 122,6 & 118,0 & 91,8 & 169,5 \\
\hline Cuenta exonerada & 169,6 & 139,2 & 196,7 & 232,8 & 209,9 & 206,6 & 178,5 & 218.1 & 143,9 & 233,6 \\
\hline Cuenta total & 254,1 & 270,5 & 298,2 & 361,4 & 327,5 & 338,9 & 301,1 & 336,1 & 235,7 & 403,1 \\
\hline Cuenta por día postalta $^{\dagger}$ & 78,4 & 98,8 & 119,0 & 124,9 & 122,1 & 125,3 & 116,1 & 167,3 & 82,4 & 144,6 \\
\hline Cuenta exonerada /cuenta total (\%) & 66,7 & 51,4 & 66,0 & 64,4 & 64,1 & 61,0 & 59,3 & 64,9 & 61,0 & 58,0 \\
\hline
\end{tabular}

* Solo se muestran las cuentas de los pacientes de alta médica no efectivizada. ${ }^{\dagger}$ Cuenta por día postalta = b/a.

El monto promedio exonerado a cada paciente con alta médica no efectivizada por falta de pago del 2001 al 2010 no sufrió cambios drásticos; el promedio de los diez años fue USD 192,88. Del mismo modo, el porcentaje promedio exonerado a cada paciente con alta médica no efectivizada por falta de pago en la década estudiada tampoco sufrió variaciones radicales, el promedio del decenio fue $61,68 \%$.

En último lugar, es conveniente referir que los pacientes con alta médica no efectivizada dejan el hospital después de un tiempo. Es en ese periodo que los familiares logran recolectar todo el dinero posible, el cual se constituye en la cuenta pagada.

\section{DISCUSIÓN}

El presente estudio demuestra la disminución en el número de pacientes con alta médica no efectivizada en el período de estudio; de los días postalta médica, y de las cuentas totales, pagadas y exoneradas. Una explicación lógica es que estos hechos se deberían al progresivo incremento de la población asegurada por la Institución Administradora de Fondos de Aseguramiento en Salud (IAFAS) pública, y de la correspondiente cobertura mediante dispositivos legales como la Ley 27660 que declara de carácter prioritario el SIS para las organizaciones de base y wawa wasis (es un programa social del estado peruano orientado a generar condiciones favorables para el desarrollo integral de niños, particularmente aquellos en situación de riesgo y en condiciones de pobreza o extrema pobreza); la Ley 28588 que incorpora al SIS a la población mayor de 17 años en situación de extrema pobreza y pobreza, y declara prioritaria la implementación del componente de salud mental en el SIS; la Ley 29695 que incorpora a los miembros del Cuerpo General de Bomberos Voluntarios del Perú como beneficiarios del SIS; el Decreto Supremo 003-2002-SA que incorpora como prioridad las prestaciones agrupadas en los componentes materno infantil, Plan $A$ del niño de 0 a 4 años, Plan B, del niño y adolescente de 5 a 17 y Plan C de gestantes; el Decreto Supremo 0182006-SA, que amplía prestaciones del SIS para la población de mototaxistas a nivel nacional dentro del Componente de Aseguramiento Semicontributivo (SISalud); y el Decreto Supremo 014-2009-SA, que dispone la afiliación gratuita de los directivos de los núcleos ejecutores en el SIS, incluyendo a sus derechohabientes.

Las personas que a inicios de la década pasada no contaban con un seguro de salud, como los pacientes con alta médica no efectivizada por falta de pago, fueron incorporados progresivamente a la IAFAS pública gracias a las directivas políticas manifiestas en los dispositivos legales referidos, hecho que ocasionó la disminución del número de pacientes con alta médica no efectivizada por falta de pago.

Tanto antiguamente como en la actualidad, las instituciones prestadoras de servicios de salud (IPRESS), como los hospitales públicos del país, tienen problemas de diversa índole que plantean grandes desafíos como los descritos por Alvarado ${ }^{(5)}$, la optimización de recursos, el monitoreo mediante indicadores de gestión, la mejora constante de los equipos y tecnologías; asimismo, el incremento de la oferta para hacer frente a la creciente demanda de salud por parte de la población, personal profesional y especializado en todas las unidades orgánicas, entre otros. 
El problema ilustrado en el presente estudio, está enfocado a los pacientes con alta médica no efectivizada por falta de pago, realidad que confirmaría que el gasto de bolsillo es un componente principal del financiamiento del sistema de salud peruano ${ }^{(2)}$. Esta forma de financiar las prestaciones de salud de la población, origina gran impacto financiero a los hospitales, como se aprecia en el periodo estudiado donde la cuenta exonerada promedio por paciente fue de USD 192,9 que representa el 61,7\% de las cuentas totales de los años considerados, hallazgo similar al descrito por García F. et al. ${ }^{(6)}$. Asimismo, en promedio, la cuenta total por paciente con alta médica no efectivizada por falta de pago representa el doble de la remuneración mínima vital nominal de cada año analizado ${ }^{(7)}$.

Desde su promulgación, la Ley 29344, Ley Marco de Aseguramiento Universal en Salud, se ha constituido en un eslabón de la cadena encaminada a la tan necesaria meta de lograr que la totalidad de la población residente en el Perú cuente con un respaldo financiero para su salud. Esta Ley se inicia en agosto de 1997 con la implementación de la estrategia social denominada Seguro Escolar Gratuito (SEG); el cual, tiempo después, se transformó en el Seguro Materno Infantil (SMI) y, finalmente, mediante la Ley 27657, Ley del Ministerio de Salud, se erigió en el Seguro Integral de Salud, cuya misión es administrar los fondos destinados al financiamiento de prestaciones de salud individual, de conformidad con la política del sector.

En julio del año 2010, se implementó el Aseguramiento Universal en Salud en Lima Metropolitana, fecha a partir de la cual, el $\mathrm{HNCH}$ ya no realiza afiliaciones de pacientes en situación de emergencia que son admitidos al hospital. Este cambio, probablemente, incidió en que pacientes que antes eran afiliados desde el momento de su internamiento en el hospital, ahora tuvieran su afiliación días después ${ }^{(8)}$, con efectos directos para la economía del hospital.

En conclusión, el estudio muestra que el alta no efectiva incrementa los costos al paciente, teniendo que ser asumido por el hospital al exonerar la cuenta, aunque este gasto disminuyó en el periodo estudiado, esto podría deberse a la implementación de directivas impartidas por la autoridad nacional de salud que tienen consecuencias en los niveles operativos hospitalarios. Ello impacta de manera positiva o negativa en las instituciones hospitalarias $y$ en los pacientes que demandan atención, de tal forma que es imprescindible generar sistemas de evaluación permanente de estas medidas, dado que si acaso se debilita la oferta (los hospitales), la demanda antes potencial y ahora efectiva sentirá insatisfacción y podría generar malestar social cuyas consecuencias pueden ser difíciles de predecir generando un contrasentido a la intencionalidad genuina de las directivas.

\section{Contribuciones de autoría}

Todos los autores participaron en el análisis e interpretación de los datos, redacción del artículo, revisión crítica y aprobaron su versión final. Además, FG y JFP realizaron el diseño del estudio, $\mathrm{JC}$ realizó el análisis estadístico, y JH y $\mathrm{AO}$ recolectaron los datos.

\section{Fuentes de financiamiento}

Autofinanciado.

\section{Conflictos de interés}

Los autores declaran no tener conflictos de interés.

\section{REFERENCIAS BIBLIOGRÁFICAS}

1. Perú, Tribunal Constitucional. Constitución Política del Perú. Lima: Tribunal Constitucional; 1993.

2. World Health Organization. World Health Statistics 2011. Ginebra, Suiza: WHO; 2011.

3. Comisión Económica para América Latina y el Caribe [Internet]. Anuario Estadístico de América Latina y el Caribe; 2010. Santiago, Chile: CEPAL/ECLAC; 2012 [citado el 01 de abril del 2012]. Disponible en: http://websie.eclac.cl/ anuario_estadistico/anuario_2010/esp/index.asp

4. Ministerio de Salud, Superintendencia Nacional de Aseguramiento en Salud. Boletín Estadístico. Primer trimestre 2011. Lima, Perú: MINSA; 2011.

5. Alvarado B, Berly A. Evaluación de Indicadores de Gestión en Hospitales Nacionales del Ministerio de Salud en Lima 1996-2001 [Tesis para optar el grado de magíster en Salud Pública]. Escuela de Post Grado, Universidad Católica Santa María. Arequipa, Perú, 2007.

6. García F, Cieza J, García H, Berly B. Los costos de las altas médicas no efectivizadas en un hospital de Lima, Perú, 2001-2002*. Rev Perú Med Exp Salud Publica. 2003;20(4):211-15. ISSN 1726-4634.

7. Instituto Nacional de Estadística e Informática [Internet]. Remuneración mínima vital entre 2001-2011. Lima, Perú: INEI; 2012 [citado el 01 de abril del 2012]. Disponible en: http://www.inei.gob.pe/biblioineipub/bancopub/Est/ Lib0988/cuadros/apurimac/2_3_3_16.xls

8. El Peruano. Ley marco de aseguramiento universal en salud. Lima: Congreso de la República; jueves 9 de abril de 2009.

Correspondencia: Félix Santiago García Ahumada

Dirección: Avenida Paseo de la Castellana 1080 Departamento 102-B; Lima 33, Perú.

Teléfono: (51) 993450400

Correo electrónico: felixgarcia@yahoo.com 